\title{
Stability of Radial Flow Subjected to a Radial Magnetic Field
}

\author{
H. A. Jasmine \\ Department of Mathematics, University of Rajshahi, \\ Rajshahi 3205, Bangladesh
}

\begin{abstract}
The linear stability of radial flow of a viscous fluid in the presence of a radial magnetic field is investigated. Basic velocity field $\mathbf{q}_{0}=(\mathrm{c} / \mathrm{r}, 0, \mathrm{~W})$ and magnetic field $\mathbf{B}_{0}=(\mathrm{A} / \mathrm{r}, 0,0)$ are considered in an annulus between two concentric cylinders. To analyze hydro-magnetic stability, inner product method is employed. The stability condition derived is found to remain valid even when the local velocity is not entirely radial, and that the magnetic field exerts a stabilizing effect on the flow.
\end{abstract}

\section{Introduction}

The linear stability of a steady nondissipative flow of an incompressible fluid between two concentric cylinders with circular streamlines was studied by Rayleigh [1] for axisymmetric disturbances. Howard and Gupta [2] examined MHD stability with axisymmetric disturbances for a steady nondissipative helical flow of a conducting fluid with velocity components $\left(0, \mathrm{~V}_{\theta}(\mathrm{r}), \mathrm{W}(\mathrm{r})\right)$ and mgnetic field either axial or azimuthal .Using a technique developed by Barston [3], Bhattacharyya et al. [4] investigated the hydro-magnetic stability for a nondissipative flow of an incompressible conducting fluid with non-axisymmetric disturbances.

Vorobev et al. [5] observed the case for low magnetic Reynolds number employing

direct numerical simulations and large eddy simulation of a forced flow in a periodic box. A series of simulations is performed with different magnetic field strengths and varying Reynolds number. Dawes [6] has shown that in the presence of a vertical magnetic field, convection may instead occur in vigorous cells seperated by regions of strong magnetic field strength. Zhang and Busse [7] investigated the instability of an electrically conducting fluid of magnetic diffusivity and viscosity in a rapidly rotating sphere when toroidal magnetic field is present. Thess and Zikanov [8] examined the robustness of two-dimensional inviscid MHD flows at low magnetic Reynolds numbers with three dimensional 
perturbations. Rajaee and Shokri [9] considered the case when a transition layer exists between two fluids, and both density and magnetic field change across this layer. The numerical calculations show that increase of the Mach number and compressibility has a destabilizing influence, the increases in magnetic field strength and density provide a stabilizing effect. Deka and Gupta [10] have analyzed linear stability of wide-gap MHD dissipative Couette flow of an incompressible electrically conducting fluid between two rotating concentric circular cylinders when a uniform axial magnetic field is present.

In this presentation, we have take the basic velocity field $\mathbf{q}=(\mathrm{c} / \mathrm{r}, 0, \mathrm{~W})$ and magnetic field $\mathbf{B}=(\mathrm{A} / \mathrm{r}, 0,0)$ in an annulus outside of which a permeable shell is placed. Following Bhattacharyya et al. [4], we have also used inner product method to deduce the stability condition. They showed that radial flow with radial magnetic field remains stable for all disturbances provided, in the undisturbed state, local Alfven's speed exceeds the local velocity everywhere in this flow. We have demonstrated that the stability condition derived remains valid even when the local velocity is not purely radial.

\section{Mathematical Formulation}

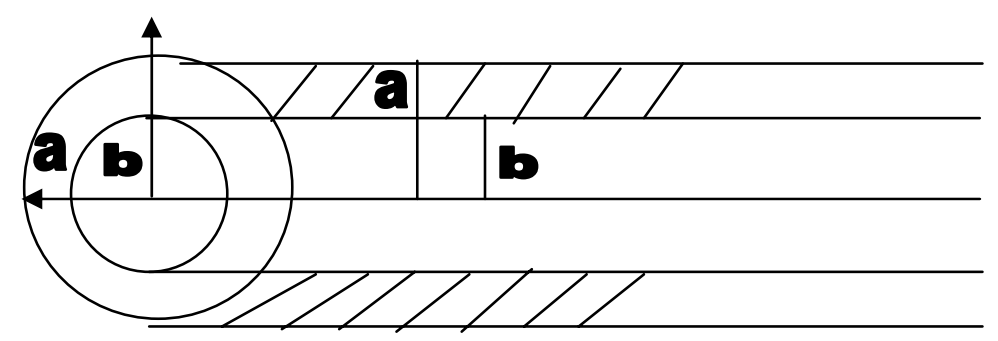

Fig1: Two porous concentric cylinders $r=a$ and $r=b(a>b)$.

Consider an inviscid incompressible flow of a perfectly conducting fluid between two porous concentric cylinders $r=a$ and $r=b(a>b)$, the flow being caused by a line source of strength $2 \pi \mathrm{c}$ per unit length, where $c$ is a constant. In a cylindrical coordinate system $(\mathrm{r}, \theta, \mathrm{z})$, the basic velocity field $\mathbf{q}_{\mathbf{0}}=(\mathrm{c} / \mathrm{r}, 0, \mathrm{~W})$, where $\mathrm{W}$ is a constant, satisfies the equation of continuity. We assume radial magnetic field $\mathbf{B}_{\mathbf{0}}=(\mathrm{A} / \mathrm{r}, 0,0)$ where $\mathrm{A}$ is a constant also satisfies $\nabla \cdot \mathbf{B}_{\mathbf{0}}=0$. According to discussion by Globe [11], the physical realization of such a radial magnetic field exists in an annulus outside of which a permeable shell is placed. 
The governing equations are

$$
\begin{aligned}
& \frac{\delta \bar{q}}{\delta t}+(\bar{q} \cdot \nabla) \bar{q}=-\frac{1}{\rho} \nabla P+\frac{\mu}{4 \pi \rho}(\nabla \times \bar{B}) \times \bar{B}, \\
& \nabla \cdot \bar{q}=0 \\
& \frac{\delta \bar{B}}{\delta t}=\nabla \times(\bar{q} \times \bar{B}) .
\end{aligned}
$$

where $\mathbf{q}$ is the velocity vector, $\mathrm{t}, \mathrm{P}, \rho, \mu$, and $\mathbf{B}$ represent time, pressure, density, magnetic permeability, and magnetic field, respectively. The perturbed velocity is $\mathbf{q}=\left(\mathrm{c} / \mathrm{r}+\mathrm{u}_{\mathrm{r}}, \mathrm{u}_{\theta}, \mathrm{W}+\mathrm{u}_{\mathrm{z}}\right)$. The perturbed magnetic field and the total presure (hydrodynamic and hydromagnetic) $\mathrm{p}$ are respectively taken as $\mathbf{B}=\left(\mathrm{A} / \mathrm{r}^{+}\right.$ $\mathrm{b}_{\mathrm{r}}, \mathrm{b}_{\theta}, \mathrm{b}_{\mathrm{z}}$ ) and $\mathrm{P} / \rho=\mathrm{p}_{0} / \rho+\mathrm{p}$, where $\mathrm{p}_{0}$ is the unperturbed total pressure. We assume that the perturbation quantities have $(\mathrm{t}, \theta, \mathrm{z})$ dependence of the form $\mathrm{e}^{\mathrm{i}(\sigma \mathrm{t}+\mathrm{m} \theta+\mathrm{kz})}$, where $\sigma=$ complex number, $\mathrm{m}=$ an integer, and $\mathrm{k}=$ real number. We linearize the equtions in the usual way and seek solutions in which all perturbation quantities $\phi$ may be written as

$$
\varphi=\hat{\varphi} e^{i(\sigma t+m \theta+k z)}
$$

The linearized momentum equations are

$$
L u_{r}+\frac{C}{r}\left(D-\frac{1}{r}\right) u_{r}+\frac{A}{4 \pi \mu \rho r}\left(D-\frac{1}{r}\right) b_{r}=-D p,
$$

$$
\begin{aligned}
& L u_{\theta}+\frac{c}{r}\left(D+\frac{1}{r}\right) u_{\theta}-\frac{A}{4 \pi \mu \rho r}\left(D+\frac{1}{r}\right) b_{\theta}=-\frac{i m p}{r}, \\
& L u_{z}+\frac{c}{r} D u_{z}-\frac{A}{4 \pi \mu \rho r} D b_{z}=-i k p,
\end{aligned}
$$

The linearized magnetic induction equations are

$$
L b_{r}+\frac{c}{r}\left(D+\frac{1}{r}\right) b_{r}-\frac{A}{r}\left(D+\frac{1}{r}\right) u_{r}=0,
$$


$L b_{\theta}+\frac{c}{r}\left(D-\frac{1}{r}\right) b_{\theta}-\frac{A}{r}\left(D-\frac{1}{r}\right) u_{\theta}=0$

$L b_{z}+\frac{C}{r} D b_{z}-\frac{A}{r} D u_{z}=0$,

where $\mathrm{L}=\mathrm{i}(\sigma+\mathrm{kW})$ and $D=\frac{d}{d r}$.

The Lagrangian displacement vector $\xi$ is defined by

$\vec{q}_{1}=\frac{\partial \vec{\xi}}{\partial t}-\vec{q}_{0} \cdot \nabla \vec{\xi}-\vec{\xi} \cdot \nabla q_{0}-\frac{c}{r} \xi_{\theta} e_{\theta}$

where $\vec{q}_{1}=\vec{e}_{r} u_{r}+\vec{e}_{\theta} u_{\theta}+\vec{e}_{z} u_{z}$ and $\vec{\xi}=\vec{e}_{r} \xi_{r}+\vec{e}_{\theta} \xi_{\theta}+\vec{e}_{z} \xi_{z}$.

From equation (5), we get

$$
\begin{aligned}
& u_{r}=L \xi_{r}+\frac{c}{r}\left(D+\frac{1}{r}\right) \xi_{r}, \\
& u_{\theta}=L \xi_{\theta}+\frac{c}{r}\left(D-\frac{1}{r}\right) \xi_{\theta}, \\
& u_{z}=L \xi_{z}+\frac{c}{r} D \xi_{z} .
\end{aligned}
$$

From equations (4) and (6), we get

$$
\begin{aligned}
& b_{r}=\frac{A}{r}\left(D+\frac{1}{r}\right) \xi_{r} \\
& b_{\theta}=\frac{A}{r}\left(D-\frac{1}{r}\right) \xi_{\theta} \\
& b_{Z}=\frac{A}{r} D \xi_{Z}
\end{aligned}
$$

Substituting for $\mathrm{u}_{\mathrm{r}}, \mathrm{u}_{\theta}$ and $\mathrm{u}_{\mathrm{z}}$ from equations (6) and for $\mathrm{b}_{\mathrm{r}}, \mathrm{b}_{\theta}$ and $\mathrm{b}_{\mathrm{z}}$ from equations (7) in equations (3), we obtain

$$
\left[L+\frac{c}{r}\left(D-\frac{1}{r}\right)\right]\left[L+\frac{c}{r}\left(D+\frac{1}{r}\right)\right] \xi_{r}-\frac{A^{2}}{4 \pi \mu \rho r}\left(D-\frac{1}{r}\right) \frac{1}{r}\left(D+\frac{1}{r}\right) \xi_{r}=-D p
$$




$$
\begin{aligned}
& {\left[L+\frac{c}{r}\left(D+\frac{1}{r}\right)\right]\left[L+\frac{c}{r}\left(D-\frac{1}{r}\right)\right] \xi_{\theta}-\frac{A^{2}}{4 \pi \mu \rho r}\left(D+\frac{1}{r}\right) \frac{1}{r}\left(D-\frac{1}{r}\right) \xi_{\theta}=-\frac{i m p}{r},} \\
& {\left[L+\frac{c}{r} D\right]\left[L+\frac{c}{r} D\right] \xi_{z}-\frac{A^{2}}{4 \pi \mu \rho r} D\left(\frac{1}{r} D\right) \xi_{z}=-i k p .}
\end{aligned}
$$

Equations (8) can be written in the matrics form as

$$
\begin{aligned}
& \left\lfloor-\lambda^{2} E+i G \kappa+H \mid \bar{\xi}=-F_{\xi},\right. \\
& \bar{\xi}=\left(\xi_{r}, \xi_{\theta}, \xi_{Z}\right)^{T},
\end{aligned}
$$$$
\text { where } \mathrm{L}=\mathrm{i} \lambda, \quad \lambda=\sigma+\mathrm{Wk} \text {, }
$$

$$
\begin{aligned}
& E=\left[\begin{array}{lll}
1 & 0 & 0 \\
0 & 1 & 0 \\
0 & 0 & 1
\end{array}\right], \quad i G=\left[\begin{array}{lll}
\frac{2 i c}{r} D & 0 & 0 \\
0 & \frac{2 i c}{r} D & 0 \\
0 & 0 & \frac{2 i c}{r} D
\end{array}\right], \\
& H=\left[\begin{array}{lll}
H_{11} & 0 & 0 \\
0 & H_{22} & 0 \\
0 & 0 & H_{33}
\end{array}\right], F_{\xi}=\left[\begin{array}{c}
D p \\
\frac{i m p}{r} \\
i k p
\end{array}\right], \\
& H_{11}=\left[c^{2}-\frac{A^{2}}{4 \pi \mu \rho}\right] \frac{1}{r}\left(D-\frac{1}{r}\right)\left[\frac{1}{r}\left(D+\frac{1}{r}\right)\right], \\
& H_{22}=\left[c^{2}-\frac{A^{2}}{4 \pi \mu \rho}\right] \frac{1}{r}\left(D+\frac{1}{r}\right)\left[\frac{1}{r}\left(D-\frac{1}{r}\right)\right], \\
& H_{33}=\left[c^{2}-\frac{A^{2}}{4 \pi \mu \rho}\right]\left[\frac{1}{r} D\left(\frac{1}{r} D\right)\right] .
\end{aligned}
$$


Here $\mathrm{E}, \mathrm{iG}$, and $\mathrm{H}$ are independent of $\lambda$. On the other hand $\nabla \cdot \mathbf{q}=0, \nabla \cdot \mathbf{B}=0$, and $\nabla . \xi=0$. Moreover

$$
\begin{aligned}
& \left(D+\frac{1}{r}\right) u_{r}+\frac{i m u_{\theta}}{r}+i k u_{z}=0 \\
& \left(D+\frac{1}{r}\right) b_{r}+\frac{i m b_{\theta}}{r}+i k b_{z}=0 \\
& \left(D+\frac{1}{r}\right) \xi_{r}+\frac{i m \xi_{\theta}}{r}+i k \xi_{z}=0
\end{aligned}
$$

According to Barston [3], the inner product can be defined as

$$
<\xi, \eta>=\int_{a}^{b}\left(\bar{\xi}_{r} \eta_{r}+\bar{\xi}_{\theta} \eta_{\theta}+\bar{\xi}_{z} \eta_{z}\right) r d r=0
$$

with the boundary conditions $\xi_{\mathrm{r}}=\xi_{\theta}=\xi_{\theta}=0$ at $\mathrm{r}=\mathrm{a}$, b. Taking inner product with $\xi$

$$
<\xi,\left[-\lambda^{2} E+i G \lambda+H\right] \xi>=<\xi, F_{\xi}>,
$$

where

$$
\left\langle\xi, F_{\xi}\right\rangle=\int_{a}^{b}\left[\xi_{r} D p+\xi_{\theta}\left(\frac{i m}{r}\right) p+\xi_{z} i k p\right] r d r=-\int_{a}^{b} \overline{\left[\left(D+\frac{1}{r}\right) \xi_{r}+\frac{i m}{r} \xi_{\theta}+i k \xi_{Z}\right]} r d r=0
$$

Hence,

$$
\lambda^{2}<\xi, E \xi>-\lambda<\xi, i G \xi>-<\xi, H \xi>=0,
$$

Where

$$
<\xi, E \xi>=\int_{a}^{b}\left[\left|\xi_{r}\right|^{2}+\left|\xi_{\theta}\right|^{2}+\left|\xi_{Z}\right|^{2}\right] r d r
$$

Hence, $<\xi, E \xi>$ is real.

$$
<\xi, i G \xi>=-2 i c \int_{a}^{b}\left[\xi_{r} D \bar{\xi}_{r}+\xi_{\theta} D \bar{\xi}_{\theta}+\xi_{Z} D \bar{\xi}_{Z}\right] d r=<\overline{\xi, i G \xi}>.
$$

Hence, $<\xi, \mathrm{iG} \xi>$ is real. 
$<\xi, H \xi>=-\left(c^{2}-\frac{A^{2}}{4 \pi \mu \rho}\right) \int_{a}^{b}\left[\frac{1}{r^{3}}\left|D\left(r \xi_{r}\right)\right|^{2}+r\left|D\left(\frac{\xi_{\theta}}{r}\right)\right|^{2}+\frac{1}{r}\left|D\left(\xi_{z}\right)\right|^{2}\right] r d r$

Hence, $<\xi, \mathrm{H} \xi>$ is real. Therefore, equation

$\lambda^{2}<\xi, E \xi>-\lambda<\xi, i G \xi>-<\xi, H \xi>=0$

is a quadratic equation in $\lambda$ with real co-efficients. From equation (15),

$\lambda=\frac{<\xi, i G \xi> \pm S^{1 / 2}}{2<\xi, E \xi>}$,

where $S=(<\xi, i G \xi>)^{2}+4<\xi, E \xi><\xi, H \xi>$.

\section{Conclusions}

\section{Case 1:}

If $\frac{A^{2}}{4 \pi \mu \rho}>c^{2}$, then $(<\xi, \mathrm{H} \xi>)>0$.

The roots $\lambda$ will be real. If $S \geq 0$ then the motion accordingly will be oscillatory.

\section{Case 2:}

If $c^{2}>\frac{A^{2}}{4 \pi \mu \rho}$, then $(<\xi, \mathrm{H} \xi>)<0$.

(i)

If $(<\xi, i G \xi>)^{2}<|4<\xi, E \xi><\xi, H \xi>|$, then $\mathrm{S}<0$ that is

$$
\lambda=\frac{<\xi, i G \xi> \pm i n^{1 / 2}}{2<\xi, E \xi>},
$$

where $\mathrm{S}=-\mathrm{n}$, and $\mathrm{n}$ is positive.

$\lambda=\lambda_{r} \pm i \lambda_{i}$

where 


$$
\begin{aligned}
& \lambda_{r}=\frac{<\xi, i G \xi>}{2<\xi, E \xi>}, \\
& \lambda_{i}=\frac{n^{1 / 2}}{2<\xi, E \xi>} .
\end{aligned}
$$

(a) The motion will be stable if $\lambda_{i}>0$ and

(b) the motion will be unstable if $\lambda_{\mathrm{i}}<0$.

(ii) If $(<\xi, i G \xi>)^{2}>|4<\xi, E \xi><\xi, H \xi>|$, then $\mathrm{S}>0$ and accordingly the motion will be oscillatory.

Therefore, we conclude that stability condition deduced above remains valid even if the local velocity is not purely radial, and that the magnetic field has a stabilizing effect on the flow.

\section{References}

1. Rayleigh, I : Scientific papers, $\underline{6}, 447$ (1920)

2. Howard, L. N and Gupta, A.S : J. Math. Anal. Appl. 106, 24 (1985)

3. Barston, E. M. : Int. J. Eng. Sci, $\underline{18}$, 447 (1980)

4. Bhattacharyya, A, Gupta, A. S and Ganguly, K: Math. S. S., 115, 51 (1985)

5. Vorobev, A, Zikanov, O, Davidson, P and Knaepen, B : Phys. Fluid, 17, 12 (2005)

6. Dawes, J. H. P: J. Fluid Mech., 570, 385 (2007)

7. Zhang, K. and Busse, F. H : J. Fluid Mech., 304, 263 (1995)

8. Thess, A and Zikanov, O: J. Fluid Mech., 579, 383 (2007)

9. Rajaee, L and Shoki, B : J. Plasma Phys., 74, 827 (2008)

10. Deka, R. K and Gupta, A.S : J. Fluids Eng.., 129, 302 (2008)

11. Globe, S : Phys. Fluid, 2, 404 (1959) 